\title{
Cancer of Unknown Primary With Presumptive Pancreatic Cancer Treated With FOLFIRINOX
}

\author{
Naonori Inoue ${ }^{\mathrm{a}, \mathrm{b}}$, Genichiro Matsumoto ${ }^{\mathrm{a}}$, Shiho Sumitomo ${ }^{\mathrm{a}}$, Atsushi Sirakawa ${ }^{\mathrm{a}}$, \\ Koichiro Mandai ${ }^{a}$, Takuji Kawamura ${ }^{\mathrm{a}}$, Koji Uno ${ }^{\mathrm{a}}$
}

\begin{abstract}
A 52-year-old man was found to have an increase in carbohydrate antigen (CA)19-9. He underwent endoscopic ultrasound/fine needle aspiration (EUS-FNA) of the soft tissue shadow near the pancreas and was diagnosed with adenocarcinoma. The cancer was judged to be of unknown primary. Cancers of unknown primary make up 3-5\% of malignant tumors, and it is difficult to determine the treatment policy. FOLFIRINOX's efficacy in cancer of unknown primary is unclear. In this case, he received chemotherapy with FOLFIRINOX. After five cycles of treatment, the best effect was stable disease (SD). However, he died about 6 months after the onset of symptoms. Pathological anatomy after death revealed that the primary lesion was in the pancreas. FOLFIRINOX may be effective for cancers of unknown primary. Pathological anatomy is important to confirm the validity of treatment. It is important to consider giving chemotherapy if there is a presumed primary tumor even if the cancer is of unknown primary.
\end{abstract}

Keywords: Cancer of unknown primary; FOLFIRINOX; Pathological autopsy

\section{Introduction}

Even with advances in diagnostic technologies, there are cases in which cancer of unknown primary is diagnosed, and it is difficult to determine the treatment policy. Treatment with a taxane- or platinum-based regimen is recommended for patients with poor prognosis for cancers of unknown primary. This case was a cancer of unknown primary, but carbohydrate antigen (CA)19-9, s-pancreas antigen-1 (SPan-1), and pancreatic cancer-associated antigen (DU-PAN-2) were abnormally high. Endoscopic ultrasound/fine needle aspiration (EUS-FNA) was

Manuscript submitted October 14, 2021, accepted October 28, 2021

Published online November 5, 2021

aDepartment of Gastroenterology, Kyoto Second Red Cross Hospital, 355-5 Haruobi-cho, Kamigyo-ku, Kyoto 602-8026, Japan

${ }^{\mathrm{b}}$ Corresponding Author: Naonori Inoue, Department of Gastroenterology, Kyoto Second Red Cross Hospital, 355-5 Haruobi-cho, Kamigyo-ku, Kyoto 602-8026, Japan. Email: inoue_ahli@yahoo.co.jp

doi: https://doi.org/10.14740/jmc3812 performed of the soft tissue shadow near the tail of the pancreas. And a pathological diagnosis of adenocarcinoma was made. Pancreatic cancer was presumed and FOLFIRINOX was performed and it was effective. Pancreatic cancer was confirmed by postmortem pathological autopsy.

\section{Case Report}

\section{Investigations}

The patient was a 52-year-old man. He had no significant medical history. His family history included colorectal cancer and prostate cancer in his father. A blood test in June 2020 revealed an abnormally high level of hepatobiliary enzymes, and he was referred to our hospital.

\section{Diagnosis}

A blood test at our hospital also showed an increase in hepatobiliary enzymes, and CA19-9, SPan-1, and DU-PAN-2 were abnormally high. Thoracoabdominal computed tomography (CT) showed soft shadows near the liver hilum, near the celiac artery, near the tail of the pancreas, omentum, ileocecal region, and appendix (Fig. 1a). No hypovascular enhancement and pancreatic duct dilatation, which are typical imaging features of pancreatic ductal adenocarcinoma, were observed. Positron emission tomography (PET)-CT showed abnormal uptake in soft tissue shadows (Fig. 1b).

EUS-FNA was performed of the soft tissue shadow near the tail of the pancreas (Fig. 2). The soft tissue was not in contact with the pancreas. And a pathological diagnosis of adenocarcinoma (cytokeratin 7 (CK7)+/CK20+/CA19-9+, and microsatellite instability (MSI)-) was made. Pancreatic head tumor was not clear when EUS was performed.

\section{Treatment}

FOLFIRINOX was started as a treatment for cancer of unknown primary, in which the clear primary lesion is unknown and pancreatic cancer is presumed. On August 18, 2020, obstructive jaundice due to an increase in the liver hilum tumor was observed. No obstruction of the lower common bile duct 

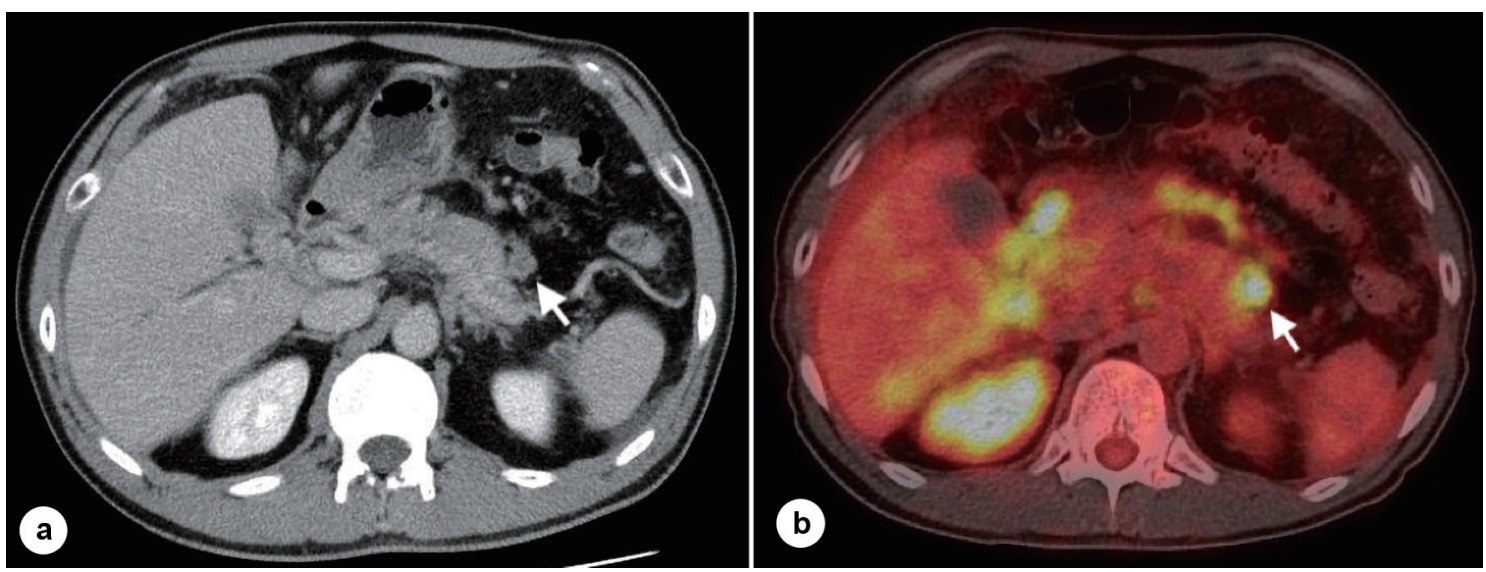

Figure 1. CT scan and PET-CT scan. (a) Intravenous contrast-enhanced CT scans show the soft tissue shadow near the tail of the pancreas (arrow). (b) PET-CT scans show the soft tissue shadow near the tail of the pancreas (arrow). CT: computed tomography; PET: positron emission tomography.

was observed. He underwent endoscopic retrograde cholangiopancreatography (ERCP), and an endoscopic retrograde biliary drainage (ERBD) tube was placed. This led to an improvement in jaundice and hepatobiliary enzyme levels. A thoracoabdominal contrast-enhanced CT performed on September 3, 2020, for the purpose of determining the effects showed SD and a reduction in ascitic fluid. A decrease in tumor markers was observed. We decided to continue treatment with FOLFIRINOX and continued it until the fifth cycle. He was subsequently admitted to the hospital on November 20, 2020, with grade 3 anemia. Abdominal CT showed exacerbation of peritoneal dissemination and increased ascites. We continued treatment with blood transfusions and ascites punctures, but the patient died on December 9, 2020.

\section{Follow-up and outcomes}

On the same day, we performed a pathological autopsy. The pathological result was a diagnosis of pancreatic head can-

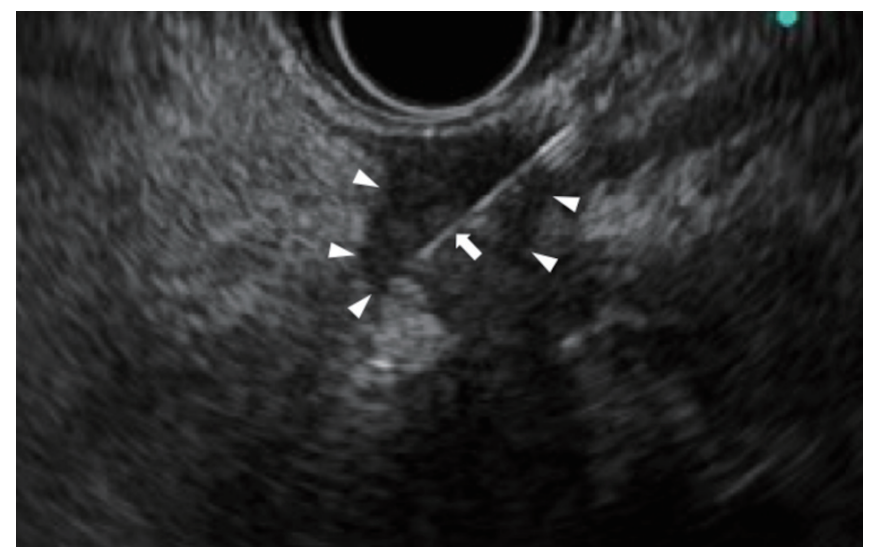

Figure 2. EUS-FNA of the soft tissue shadow near the tail of the pancreas. EUS showing the soft tissue shadow near the tail of the pancreas (arrow head) is punctured by the needle (arrow). EUS-FNA: endoscopic ultrasound/fine needle aspiration. cer (CK7+/CK20+/CA19-9+, Fig. 3). Pancreatic head cancer could not be found on EUS.

\section{Discussion}

There were two important findings in this case: FOLFIRINOX was effective against cancer of unknown primary presumed to be pancreatic cancer. Pathological anatomy is important to confirm the validity of treatment. We discuss each of these separately below.

\section{FOLFIRINOX is effective against cancers of unknown primary presumed to be pancreatic cancer}

Cancers of unknown primary make up 3-5\% of malignant tumors, and no improvement in overall survival has been observed over the last 10 years [1]. If the patient is judged to have a poor prognosis based on the location of the lesion and clinical/pathological characteristics, chemotherapy is the main treatment. Among the cancers of unknown primary in the poor prognosis group, several studies have reported on adenocarcinoma treated with a taxane- or platinum-based regimen [2, 3]. Lee et al performed an analysis that combined a systematic review and meta-analysis. Adjusted prognostic factors showed no significant difference in survival benefit between platinumbased and non-platinum-based regimens [4]. A systematic review by Amela et al concluded that there is no specific regimen that can be recommended for these cancers [5]. Currently, chemotherapy for patients with disseminated metastasis is limited to patients with good performance status (PS). The regimens in their study were often taxane- or platinum-based, but no specific regimen was recommended.

In the present case, EUS-FNA was used to collect tissue from the tail of the pancreas, and immunostaining revealed CK7+/CK20+/CA19-9+, and a blood test showed abnormal CA19-9, SPan-1, and DU-PAN-2. Since the values were high, we decided to start treatment for a suspected pancreatic cancer 


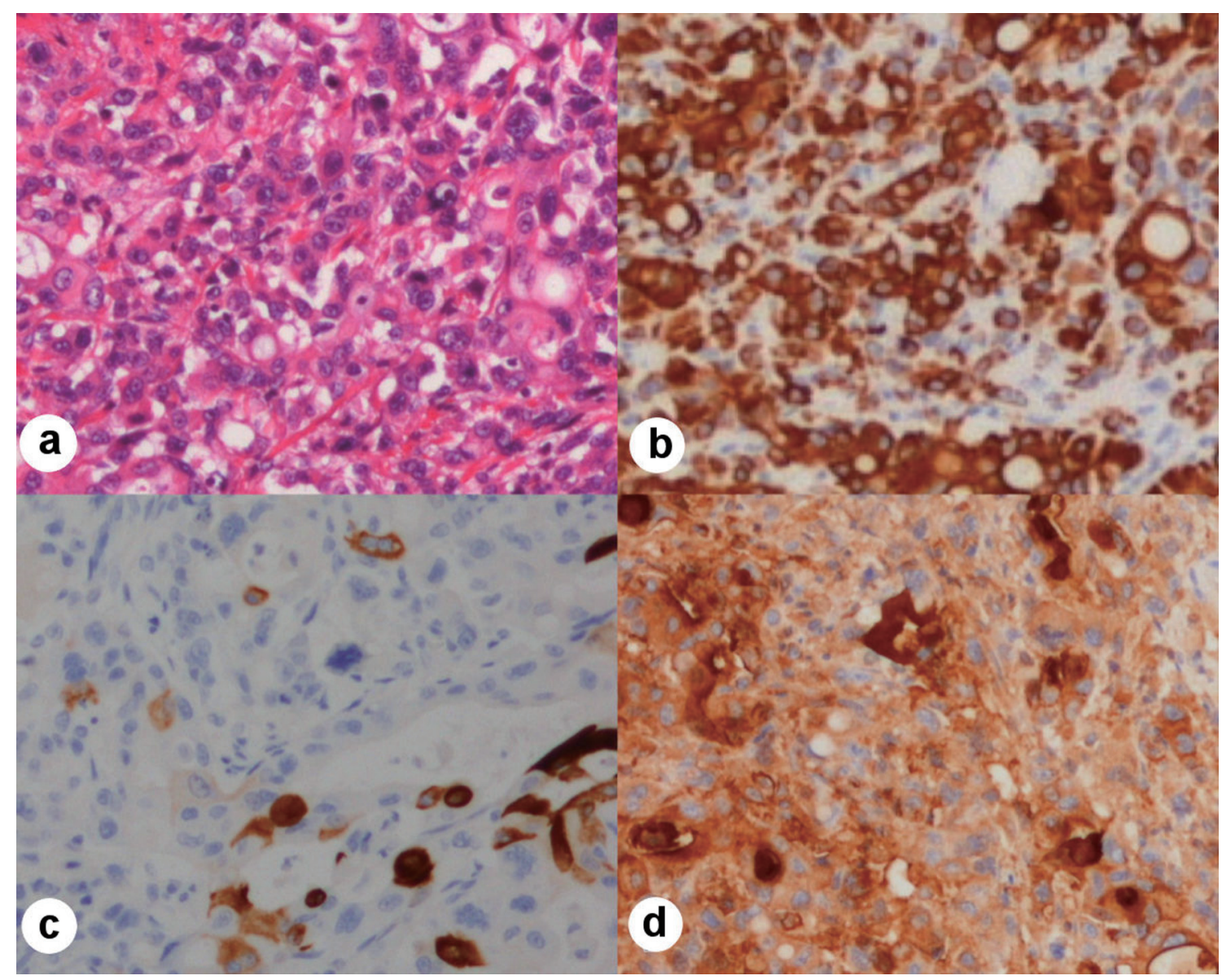

Figure 3. Neoplastic cells, hematoxylin-eosin stain, immunohistochemical stains $\times 100$. (a) Hematoxylin-eosin staining of neoplastic cells. (b) Immunohistochemical stain of CK7 showing positivity in neoplastic cells. (c) Immunohistochemical stain of CK20 showing positivity in neoplastic cells (scattered). (d) Immunohistochemical stain of CA19-9 showing positivity in neoplastic cells. CK: cytokeratin; CA: carbohydrate antigen.

of unknown primary cancer. FOLFIRINOX was selected as the regimen. A total of five cycles of treatment were performed. Although CT indicated SD, we judged that the disease condition was temporarily suppressed. Anne et al reported a case of cancer of unknown primary suspected to be pancreatic cancer treated with FOLFIRINOX [6]. This is the second report of FOLFIRINOX for cancer of unknown primary origin with presumptive pancreatic cancer. Currently, next-generation sequencing (NGS) for cancers of unknown primary is being performed, and it has been pointed out that it may improve the prognosis [7]. In the future, further optimization of treatment is desired by accumulating cases.

\section{Pathological anatomy is important to confirm validity of treatment}

In some cases, as in this case, the primary lesion cannot be identified even if cancer is diagnosed by pathological examination of the metastatic lesion. Cancers of unknown primary make up 3-5\% of all malignancies. The prognosis for cancers of unknown primary is poor and overall survival is 6 months
[1]. There is no commonly agreed-upon chemotherapy regiment for such cancers. In order to confirm the validity of treatment to improve future treatment, it is important to examine the primary lesion by pathological autopsy. In this case, the primary lesion could be confirmed in the head of the pancreas by performing pathological autopsy. The primary lesion in this case could not be confirmed by contrast-enhanced CT or PET-CT while the patient was alive. FOLFIRINOX is not a common treatment for cancers of unknown primary. However, in this case, treatment with FOLFIRINOX was considered to be appropriate.

In this case, pancreatic cancer was diagnosed as a cancer of unknown primary, and treatment was performed in the same manner as for pancreatic cancer. Later, pathological autopsy revealed pancreatic cancer. It is not always necessary to choose taxane- or platinum-based chemotherapy, which has been widely reported on, even for cancers of unknown primary. If there is a suspected primary lesion, the physician may choose the treatment most appropriate for cancers of that origin. In the case of cancer of unknown primary, it is important to perform pathological autopsy as much as possible in order to confirm the validity of the treatment performed. 
This will allow the physician to improve future treatment for cancer patients.

\section{Acknowledgments}

None to declare.

\section{Financial Disclosure}

None to declare.

\section{Conflict of Interest}

None to declare.

\section{Informed Consent}

The patient described in the case report had given informed consent for the case report to be published.

\section{Author Contributions}

Naonori Inoue: case selection, discussion and drafting manuscript; Genichiro Matsumoto: drafting manuscript and revision; Shiho Sumitomo: drafting manuscript and revision; Atsushi Sirakawa: manuscript revision and final approval; Koichiro Mandai: manuscript revision and final approval; Takuji Kawamura: manuscript revision and final approval; Koji Uno: case selection, planning, manuscript revision and final approval.

\section{Data Availability}

The data supporting the findings of this study are available from the corresponding author upon reasonable request.

\section{Abbreviations}

CA: carbohydrate antigen; EUS-FNA: endoscopic ultrasound/ fine needle aspiration; SPan: s-pancreas antigen; DU-PAN-2: pancreatic cancer-associated antigen; CT: computed tomography; PET: positron emission tomography; CK: cytokeratin; MSI: microsatellite instability; ERCP: endoscopic retrograde cholangiopancreatography; ERBD: endoscopic retrograde biliary drainage; SD: stable disease; PS: performance status; NGS: next-generation sequencing

\section{References}

1. Losa F, Soler G, Casado A, Estival A, Fernandez I, Gimenez S, Longo F, et al. SEOM clinical guideline on unknown primary cancer (2017). Clin Transl Oncol. 2018;20(1):89-96.

2. Hainsworth JD, Spigel DR, Clark BL, Shipley D, Thompson DS, Farley C, West-Osterfield K, et al. Paclitaxel/ carboplatin/etoposide versus gemcitabine/irinotecan in the first-line treatment of patients with carcinoma of unknown primary site: a randomized, phase III Sarah Cannon Oncology Research Consortium Trial. Cancer J. 2010;16(1):70-75.

3. Gross-Goupil M, Fourcade A, Blot E, Penel N, Negrier S, Culine S, Chaigneau L, et al. Cisplatin alone or combined with gemcitabine in carcinomas of unknown primary: results of the randomised GEFCAPI 02 trial. Eur J Cancer. 2012;48(5):721-727.

4. Lee J, Hahn S, Kim DW, Kim J, Kang SN, Rha SY, Lee $\mathrm{KB}$, et al. Evaluation of survival benefits by platinums and taxanes for an unfavourable subset of carcinoma of unknown primary: a systematic review and meta-analysis. Br J Cancer. 2013;108(1):39-48.

5. Amela EY, Lauridant-Philippin G, Cousin S, Ryckewaert T, Adenis A, Penel N. Management of "unfavourable" carcinoma of unknown primary site: synthesis of recent literature. Crit Rev Oncol Hematol. 2012;84(2):213-223.

6. Anne M, Ahmad N, Lee P, Aziz M, Lebowicz Y. An unusual presentation of isolated leptomeningeal disease in carcinoma of unknown primary with pancreatic features. J Investig Med High Impact Case Rep. 2013;1(2):2324709613494830.

7. Adashek JJ, Kato S, Gumas S, Lee S, Okamura R, Sicklick J, Kurzrock R. 86MO Personalized molecularly matched therapies for carcinomas of unknown primary is associated with improved outcomes. Annals of Oncology. 2020;31:S275-S276. 